eye (right) was higher and " larger" than its fellow at birth.* The protrusion of the right eye had gradually increased, more so within the last two years, but had never been attended with any pain. The symptoms, on examination, were great protrusion of the organ, strabismus convergens, and dilatation of the pupil ; the upper eyelid was greatiy swollen, covered with large, tortuous, livid vessels, and hung in front of the eye, covering it to a considerable extent; the conjunctiva generally, but in particular of the lids, was inflamed and vascular, and protruded forwards on the lower and outer side, so as to be seen even without depressing the lower lid. The most particular point, however, was, that the structures, as high as the orbital edge superiorly, were, as well as the lid, swollen, congested, and marked by varicose vessels. When I speak of the vascularity reaching only to the upper edges of the tarsal cartilages (see former case), I do not mean that it terminates there abruptly, but only that it does not extend much higher. It was true strabismus, not lascitas, as the patient, when directed, could turn the eye outwards completely, and the external circumscribed swelling presented, on examination, no feel of a defined tumour, but only a soft puffy swelling. The eye itself was unnaturally tense and resisting, not nearly so much as in hydrophthalmia, resulting, as I have before explained, from the same force which protrudes the globe ; in appearance, there was nothing unnatural ; the sclerotica was sound, and of a healthy colour; the iris not forced forwards; the cornea of its natural convexity, size, and transparency ; the chambers normal ; the pupil dilated and sluggish in its movements.

The child complained of nqpain in the eye or head; its vision was defective, but by no means lost; and its health had been pretty good since birth. The strabismus probably resulted not from pressure, but from the defect of vision interfering with the accurate use of the other eye. This case requires no comment; in it were seen all the symptoms peculiar to exophthalmia, characteristically proving the truth of $\mathrm{my}$ observations, and clearly diagnostic from those of hydrophthalmia.

The thirty-second, and last, division treats of the presence of a circumscribed tumour displacing and fixing the globe. It behoves us, in every doubtful case, to examine the nature of the swelling, and ascertain its uniformity, since the majority of cases attended with exophthalmic protrusion are occasioned by a circumscribed tumour, or a

* All eyes in individuals of the same period of life are nearly of the same size, their apparent magnitude depending on the extent to which the organs are uncovered by the lids; hence, the more uncovered the larger they appear. tumour larger at one than another part. When such is evident to the eye, there can be no doubt about the matter; but in the early stages of the affection, we may be unable to discover its existence by the eye alone, although present, and forcing the eyeball in the opposite direction. This may be accounted for by the comparatively small size of the swelling, and the fact of its being involved in the surrounding tumefaction. But the touch may here make manifest what the sight, unaided, either proved inefficient to discover, or suggested doubts to the mind of the observer-the part at which the protruding growth will ultimately show itself feels, by careful manipulation, elevated and resisting-it conveys the idea of some hard circumscribed growth imbedded in the surrounding soft textures. This is precisely the exact state of parts, and is therefore truly diagnostic.

Such a tumour displaces the globe in the opposite direction: thus, in the case just mentioned, the tumour existed at the lower and outer part of the orbit, whilst the globe was ordinarily turned upwards and inwards, not, however, being fixed in this patient; indeed, as I have before remarked, it was doubtful whether it resulted from pressure, or from the defect of vision. When very slight, the protruded eyeball would be turned out of its axis but inconsiderably, and its motions limited to but a slight degree; yet this proposition would always hold goodnamely, that the protrusion would be most considerable in the opposite direction to the siluation of the expelling force. And hence that the presence of a circumscribed tumour, discoverable by the united powers of touch and vision, displacing and comparatively fixing the eyeball, may with advantage be enumerated among the diagnostic symptoms of hydrophthalmia and exophthalmia. In my next paper, I trust to detail a case of hydrophthalmia, evidently of inflammatory origin, to which the term chronic hyaloiditis may be applied.

\section{ABSCESS OF THE MAMMARY GLAND.}

\section{To the Editor of THE LANCET.}

SIR :-In your report of the meeting of the Medical Society of London, ou Monday, Mirch $15 \mathrm{hh}, I$ observe that Mr. Kingdon "drew the attention of the society to a case of abscess, situated in the tissues beneath the mammary gland; it had been treated as a common milk abscess ; and when the matter had pointed in one place, and an incision was made into the breast, it subsequently formed in various parts of the gland; and although everything that medical aid could do was done, the patient sunk. After death an extensive abscess was found situated behind the gland, through which the matter had burrowed in various directions; the 
gland itself was otherwise healthy. In another case, in which he had lalely been consulted, a hard, painful tumour was situ. ated behind, and totally unconnected with the mammary gland. Under proper remedies the pain was much relieved, but subsequently returned; and although there was no symptom of scirrhus present, he had given his consent to the removal of the breast, recollecting, as he did, the fearful consequences following the former case, and that the operation would not be likely to be a serious one." On referring to my notes, $I$ find the following remarks on a disease which, I apprehend, must be the same as that which Mr. Kingdon describes. If you think them worth publication, I shall be obliged by your inserting them in your next Number.

There is a disease of the breast differing from the common milk abscess, which must, I apprehend, be of rare occurrence, having seen only four or five examples in the course of my life. All the cases which have fallen under my observation have taken place dur. ing suckling, and generally soon after delivery. It does not exactly correspond to the description of the deep-seated abscess mentioned in Mr. Hey's book, the cavities of which were filled with a soft, purple fungus, althongh, in other respects, it nearly resembles it, and both are cured by the same plan of treatment. This abscess forms in and behind the mammary gland, more slowly and with less pain than the common milk abscess; it does not come to the surface so soon, neither is the skin so much inflamed, or the fluctuation so distinct, consequently it is not opened early, but burrows behind and around the gland, breaking in several places, and forming sinuses in a variety of directions, none of which show a disposition to heal. The usual treatment is unavailing in these cases; a great discharge is constantly kept up, which soon affects the general health, and reduces tbe patient to a state of great debility, which I believe would destroy her, unless proper means were resorted to, to arrest the disease. The first case of the kind which fell under my care was a lady, who had been confined three months, and was much exhausted by pain and constant discharge. The breast, which was naturally full and fleshy, was not much increased in size, but had an unhealthy ap. pearance, with several openings leading to deep sinuses communicating with each other in various directions. At this time I was not aware of the obstinate nature of the disease, and endeavoured to heal these by injections of sulphate of zinc, stimulating friction, and pressure evenly applied with plaster and bandage; all my efforts were ineffectual, and $I$ was under the necessity of dividing all the sinuses, which ran through the whole the substance of the breast, so that it was literally cut into strips. The operation was a serious one, and attended with considerable hæmorrhage; but the good effects were presently apparent, for as soon as my patient had recovered from the immediate shock of the operation, the wounds put on a healthy aspect; she rapidly got better, and recovered perfectly in six weeks. At her next confinement she came from a considerable distance to place herself under my care; and as there was no hope of her ever being able to give suck again in that breast, I covered it carefully with a dia. chylon plaster as soon as she was delivered, which completely suspended the secretion of milk in that breast, although she was able to nurse as well as usual in the other, and she passed through her confinement without the least inconvenience. Since that time she has had several other children, and uniformly adopted the same course with the like success.

I have met with three other cases, which were treated in the like manner with the same result. I was consulted on another case, in which the patient refused to sub. mit to the plan proposed, and tried various remedies under different practitioners, but was at last obliged to have the same operation performed. If I saw one of these cases in an early stage, I should not hesitate to make a free opening the instant the forma. tion of matter could be ascertained, in the hope of preventing so painful and severe an operation.

$$
\text { Bridgewater, April 12, } 1841 \text {. }
$$

Jonathan Toogood,

\section{ON THE}

\section{PROPER TREATMENT OF THE INSA NE.}

\section{To the Editor of THE LANCET.}

SrR :-Being a medical superintendent of a county asylum, I have not been an uninte. rested reader of the many cases which have been published in your Journal on the subject of the treatment of the insane; nor have the conflicting opinions on the so-called hamane system been unregarded by me: controversy is not my forte, nor would $I$ have encountered the chance of being engaged in it but from a conviction, after perusing the letter in your Journal of the 2 th of March last, signed "A Medical Superintendent," that it is calculated to mislead your readers on the subject of which it treats. I am quite as anxious as he or any other of my com. peers, that the treatment and management of the insane should be conducted on the most unbounded scale of kindness and bevevo. lence, and I give my professional bretbren similarly engaged full credit for entertaining the same views. I confess, therefore, that it was matter of surprise to me, when I found that the writer of the letter alluded to should have conveyed a censure by implication on the superintendents of English asylums, when 